\title{
Safety, efficacy, and life satisfaction following epilepsy surgery in patients aged 60 years and older
}

\author{
Sandra Dewar, RN, MS, ${ }^{1}$ Dawn Eliashiv, MD, ${ }^{1}$ Patricia D. Walshaw, PhD, ${ }^{2}$ \\ Jerome Engel Jr., MD, PhD, ${ }^{1}$ Itzhak Fried, MD, PhD, ${ }^{3}$ and Brian D. Moseley, MD ${ }^{4}$
}

Departments of ${ }^{1}$ Neurology, ${ }^{2}$ Psychiatry and Biobehavioral Sciences, and ${ }^{3}$ Neurosurgery, David Geffen School of Medicine at UCLA, Los Angeles, California; and ${ }^{4}$ Department of Neurology and Rehabilitation Medicine, University of Cincinnati, Ohio

\begin{abstract}
OBJECTIVE Despite its potential to offer seizure freedom, resective epilepsy surgery (RES) is seldom performed in patients 60 years of age or older. Demonstrating successful outcomes including an improved quality of life may raise awareness about the advantages of referring this underrepresented population for specialized evaluation. Accordingly, the authors investigated outcomes and life fulfillment in patients with an age $\geq 60$ years who had undergone RES.

METHODS All patients who, at the age of 60 years or older, had undergone RES for medically refractory focal onset seizures at the authors' center were evaluated. A modified Liverpool Life Fulfillment (LLF) tool was administered postoperatively (maximum score 32). Seizure outcomes were classified according to the Engel classification system.

RESULTS Twelve patients underwent RES. The majority of patients (9 [75\%] of 12) had at least 1 medical comorbidity in addition to seizures. The mean follow-up was $3.1 \pm 2.1$ years. At the time of the final follow-up, $11(91.7 \%)$ of 12 patients were documented as having a good postsurgical outcome (Engel Class I-II). Half (6 of 12 patients) were completely seizure free (Engel Class IA). Liverpool Life Fulfillment (LLF) data were available for 11 patients. Following surgery, the mean LLF score was $26.7 \pm 6$. Eight patients $(72.7 \%)$ noted excellent satisfaction with their RES, with $5(45.5 \%)$ noting postoperative improvements in overall health.

CONCLUSIONS Resective epilepsy surgery is safe and effective in patients with an age $\geq 60$ years. Over $90 \%$ had a good surgical outcome, with $50 \%$ becoming completely seizure free despite 1 or more medical comorbidities in the majority. The study data indicated that an advancing age should not negatively influence consideration for RES.

http://thejns.org/doi/abs/10.3171/2015.3.JNS142317
\end{abstract}

KEY WORDS epilepsy surgery; older patients; outcomes; quality of life

$\mathrm{A}$ PPROXIMATELY 1 in 3 patients with epilepsy will not achieve seizure freedom with antiseizure medications alone. Uncontrolled seizures are estimated to account for $80 \%$ of the cost of epilepsy in the United States. ${ }^{2}$ For patients with drug-resistant focal onset seizures, resective epilepsy surgery (RES) is a superior alternative to continued antiseizure drug trials. ${ }^{9}$ Such an intervention, which can be effective at eliminating seizures in over $70 \%$ of carefully selected patients, has the potential to significantly lower seizure-related morbidity and mortality and improve quality of life (QOL). ${ }^{9}$

Approximately $25 \%$ of patients with epilepsy are estimated to have an age $\geq 60$ years. ${ }^{17}$ The highest age-specific incidence of epilepsy in industrialized countries is in those 60 years and older. ${ }^{14}$ It is anticipated that as the popula- tion ages, the need to consider surgery for older people with epilepsy will increase. Professional consensus on the optimum age for RES is lacking. Early sources considered the optimal age to be between 12 and 30 years. ${ }^{4}$ After the second Palm Desert Conference regarding RES in 1992, it was agreed that no upper age limit for RES should be set. However, it was believed that surgery in adults between the ages of 45 and 60 should be approached with caution. At the time of the conference, very few centers were performing RES in patients older than 60 because of perceived risks. ${ }^{8}$ A small series of peer-reviewed papers addressing surgical outcomes in patients over the age of 45 years show that surgical outcomes and complication rates in older versus younger populations do not appear to be significantly different. ${ }^{3,4,7,15,16}$

ABBREVIATIONS AMTL = anteromesial temporal lobe; EEG = electroencephalography; LLF = Liverpool Life Fulfillment; $Q O L=$ quality of life; RES = resective epilepsy surgery; UCLA = University of California, Los Angeles.

SUBMITTED October 8, 2014. ACCEPTED March 30, 2015

INCLUDE WHEN CITING Published online September 18, 2015; DOI: 10.3171/2015.3.JNS142317. 
Such preliminary results suggest that some degree of prejudice may exist with regard to RES in older patients. Patients over age 60 may be less likely to be offered surgery due to misperceptions including: 1) the fear that older patients have higher incidences of comorbid medical conditions, which reduce the safety of surgery; 2) the association of an older age with a longer duration of epilepsy and therefore a lower likelihood of seizure freedom following RES; 3) concerns for postsurgical cognitive decline; and 4) the possibility that older patients may be less adaptable and, consequently, that seizure freedom following RES will be less likely to increase QOL.

We report on our experience with epilepsy surgery in patients over 60 years of age with focal onset epilepsy with regard to seizure freedom and life satisfaction after surgery. We sought to determine if an upper age limit should be imposed on surgical candidates with refractory focal onset seizures. We also sought to determine the factors motivating older patients to consider RES and the impact of surgery on life fulfillment.

\section{Methods \\ Patient Selection}

Patients over 60 years of age at the time of RES were identified from the University of California, Los Angeles (UCLA), surgical series database for the period from 1998 to 2013. Retrospective chart review established comorbidities at the time of surgery, surgery side, type of surgery, and postresection pathology. We also calculated Charlson Comorbidity Index scores and 10-year survival probabilities (using those scores) for each patient. ${ }^{5,6} \mathrm{~A}$ minimum of 1 year of postoperative follow-up was required for study inclusion.

\section{Presurgical and Postsurgical Evaluations}

Presurgical evaluation of potential epilepsy surgery patients at UCLA includes continuous inpatient video electroencephalography (EEG) monitoring, MRI, FDG-PET, and (for potential temporal lobectomy candidates) the intracarotid sodium amobarbital procedure (Wada testing). Patients also typically undergo extensive neuropsychological testing, although such testing is not routinely performed in all patients following RES. None of the patients in this study underwent intracranial EEG recordings.

Following completion of the above workup, patients are presented at a multidisciplinary consensus epilepsy surgery conference attended by epileptologists, epilepsy surgeons, neuropsychologists, neuroradiologists, and nurse specialists. All available data are reviewed to determine the suitability of individual patients for RES and/or the need for further testing (for example, ictal SPECT and/ or magnetoencephalography). Patients are subsequently referred for RES if all information is congruent and there is a realistic expectation of seizure freedom and/or substantial seizure reduction from surgery in the absence of significant side effects or risks (for example, memory and/ or language impairment). At our institution no predetermined baseline cognitive level of performance is required to undergo RES. Histories of psychological disorders such as depression and/or anxiety do not necessarily exclude consideration for surgery. However, we require that those disorders be adequately treated at the time of RES. If there is any doubt about the adequate treatment of comorbid psychological disorders, a formal psychiatry evaluation and clearance are requested prior to RES.

Postoperative complications were extracted from the electronic medical record. A telephonic interview was conducted to determine seizure outcome. Outcome was classified according to the Engel classification system, ${ }^{8}$ and Engel Classes I and II were considered good outcomes.

\section{Reasons for Undergoing RES and Postsurgery Life Fulfillment}

Patients were asked about changes in health status, driving, and working after surgery. Motivation to consider surgery and reasons for delay were evaluated. The influence of seizure-related falls and injuries on decision making was assessed. Patients were also queried about falls after surgery. Patients were asked to describe what they believed to be their greatest challenge in facing RES. Telephonic interviews (conducted by S.D.) were guided by a structured questionnaire assessing the motivation to consider surgery, seizure freedom, and satisfaction with surgery. A modified Liverpool Life Fulfillment (LLF) tool was administered postoperatively to measure life changes in the spheres of health, aspects of postsurgical adjustment, and perceptions of health status following surgery. This scale was modified to an 8-question scale assessing patient perception of life fulfillment. ${ }^{21}$ We excluded workrelated items in our scale given that many patients were already retired at the time of RES and did not return to work after surgery; therefore, the maximum possible score on our modified LLF tool was 32 (Table 1).

\section{Statistical Analysis}

Data entry and statistical analysis were performed using IBM SPSS Statistics Version 19 (IBM Corp.). We used the Wilcoxon rank-sum test with continuity correction. A $\mathrm{p}$ value $<0.05$ was considered statistically significant.

\section{Results}

\section{Demographics}

Twelve patients ( 9 females and 3 males) with an age $\geq$ 60 years underwent RES during the study period. These 12 represented approximately $3 \%$ of adult RES cases during the study years. The majority of patients $(9$ [75\%] of 12) were offered a standard anteromesial temporal lobe (AMTL) resection (8 right-sided and 1 left-sided). One patient underwent a left temporal lesionectomy sparing the mesial structures. One patient underwent a right temporal lesionectomy. One case involved the resection of a right frontal lesion. Pathology revealed focal cortical dysplasia in 1 patient $(8.3 \%$, including the 1 patient who underwent a right frontal lesionectomy), cavernous hemangiomas in 2 (13.3\%), dual pathology (focal cortical dysplasia and mesial temporal sclerosis) in 1 (8.3\%), astrocytoma/low-grade glioma in $2(13.3 \%)$, and nonspecific gliosis in $5(41.7 \%)$. Mean age at the time of RES was $65 \pm 4.2$ years (range 60-74 years). For the 11 patients who underwent temporal resections, the mean age was $65 \pm 4.4$ years. Mean age at 


\section{TABLE 1. Modified LLF tool}

We would like to know how satisfied you are with your own life situation. For each question below, please select the number that best shows how you feel.

a) How satisfied are you, in general, with your family life?

Very satisfied (4 points), Satisfied (3 points), Dissatisfied (2 points), Very dissatisfied (1 point)

b) How happy do you feel about the number of close friends you have, that is, friends you feel you can confide in?

Very happy (4 points), Fairly happy (3 points), Not very happy (2 points), Not at all happy (1 point)

c) How satisfied are you, in general, with the relationship you have with your spouse/partner?

Very satisfied (4 points), Satisfied (3 points), Dissatisfied (2 points), Very dissatisfied or does not apply-no spouse/partner (1 point)

d) How much do you feel able to do the things you enjoy in your spare time?

Often (4 points), Sometimes (3 points), Rarely (2 points), Never (1 point)

e) How satisfied are you, in general, with your social life?

Very satisfied (4 points), Satisfied (3 points), Dissatisfied (2 points), Very dissatisfied (1 point)

f) How would you describe your health now?

Excellent (4 points), Good (3 points), Fair (2 points), Poor (1 point)

g) How happy are you with the way you feel about yourself?

Very happy (4 points), Fairly happy (3 points), Not very happy (2 points), Not at all happy (1 point)

h) How satisfied are you with your present standard of living?

Very satisfied (4 points), Satisfied (3 points), Dissatisfied (2 points), Very dissatisfied (1 point)

seizure onset was $38.2 \pm 13.8$ years (range $21-61$ years). It was slightly higher $(39.7 \pm 13.4$ years $)$ for the 11 patients with temporal lobe epilepsy. The mean duration of epilepsy prior to surgery was $26.9 \pm 15.2$ years (range $1-48$ years) for the entire group. This parameter increased to $31.9 \pm 10.7$ years (range $8-48$ years) for the 10 nonneoplastic patients. It was slightly lower ( $25.4 \pm 14.9$ years) for the 11 patients who underwent temporal resections.

One death due to an astrocytoma was recorded in the group. This patient died 20 months after surgery. However, at the time of death, he was free of disabling seizures following a right AMTL resection. Consequently, life satisfaction scores were calculated for the 11 remaining patients (Table 2).
One patient with a brief history of refractory seizures due to a confirmed astrocytoma (WHO Grade III) underwent a right AMTL resection within 1 year of seizure onset. In the remaining 10 cases, in which there were delays to surgery, the reasons for not considering surgical therapy earlier were recorded: never being offered surgery ( 5 [50\%]), fear of surgery and denial about severity of seizures (4 [40\%]), and failure of the first RES and low confidence in the success of repeat RES (1 [10\%]).

\section{Preoperative Neuropsychological Testing}

Nine of the 12 patients in our cohort had available neuropsychological testing prior to RES. Full neuropsychological testing data for these 9 patients are available in

TABLE 2. Outcomes following RES in older patients

\begin{tabular}{cccccccccc}
\hline $\begin{array}{c}\text { Patient } \\
\text { ID }\end{array}$ & $\begin{array}{c}\text { Age at } \\
\text { Op (yrs) }\end{array}$ & $\begin{array}{c}\text { Type of } \\
\text { Op }\end{array}$ & $\begin{array}{c}\text { Length of } \\
\text { Hospital Stay } \\
\text { (days) }\end{array}$ & $\begin{array}{c}\text { FU } \\
\text { (yrs) }\end{array}$ & Pathology & $\begin{array}{c}\text { Engel Class } \\
\text { Outcome }\end{array}$ & LLF Score & $\begin{array}{c}\text { i1 Comorbidity at } \\
\text { Time of Op }\end{array}$ & $\begin{array}{c}\text { On AEDs at } \\
\text { Final FU }\end{array}$ \\
\hline A & 60 & LAMTL & 4 & 7.5 & CG & IA & 30 & N & Y \\
\hline B & 69 & RAMTL & 6 & 6.5 & LGG & IID & 10 & Y & Y \\
\hline C & 63 & RAMTL & 4 & 3.4 & CG & IID & 31 & Y & Y \\
\hline D & 74 & RAMTL & 4 & 2 & CG & IA & 27 & Y & Y \\
\hline E & 62 & RAMTL & 4 & 1.5 & CG & III & 25 & N & Y \\
\hline F & 69 & RAMTL & 3 & 2.2 & CD+CG & IA & 25 & Y & Y \\
\hline G & 64 & RAMTL & 5 & 1.7 & Glio & IC & Deceased & Y & Deceased \\
\hline H & 68 & RT les & 4 & 3.7 & CM & IA & 31 & N & N \\
\hline I & 62 & RAMTL & 3 & 4 & Multi & IB & 30 & Y & Y \\
\hline J & 63 & LT les & 3 & 1.3 & CM & IA & 31 & Y & Y \\
\hline K & 65 & RF les & 3 & 2 & CD & IA & 27 & Y & Y \\
\hline L & 61 & RAMTL & 9 & 1 & Astro & IB & 27 & Y & Y \\
\hline
\end{tabular}

$\mathrm{AED}=$ antiepilepsy drug; Astro = astrocytoma (WHO Grade III); $\mathrm{CD}=$ cortical dysplasia; $\mathrm{CG}=$ Chaslin's gliosis; $\mathrm{CM}=$ cavernous malformation; FU = follow-up; Glio = glioblastoma; LAMTL = left AMTL; LGG = low-grade glioma; LT les = left anterior temporal lesionectomy; Multi = CG, hippocampal sclerosis, focal neuronal disorganization, and mild amyloid angiopathy; $\mathrm{N}=$ no; RAMTL = right AMTL; RF = right frontal lesionectomy; $\mathrm{RT}$ les = right temporal cavernous angioma lesionectomy; $\mathrm{Y}=$ yes. 
Table 3. No patients met the criteria for intellectual disability or dementia. Only 1 patient reported a history of depression, which was being treated at the time of RES. Four other patients reported histories of anxiety disorders. No other diagnosed psychological comorbidities were present in our cohort.

Postoperative neuropsychological testing is not standard at our institution. No patient in our cohort underwent further neuropsychological testing following RES.

\section{Seizure Outcome}

Patients were followed up for a mean of $3.1 \pm 2.1$ years (range 1-7.5 years). This duration was nearly identical (3.2 \pm 2.2 years) for the 11 patients with temporal lobe epilepsy. At the time of the final follow-up, 11 (91.7\%) of 12 patients were documented as having a good postsurgical outcome (Engel Class I-II), including 10 (90.9\%) of the 11 patients who had undergone temporal resections. Half (6/12) of all patients were completely seizure free (Engel Class IA), including 5 (45.5\%) of the 11 patients with temporal lobe epilepsy. Two patients (16.7\%) were free of disabling seizures only (Engel Class IB), while $1(8.3 \%)$ had a few early seizures but then became seizure free for over 2 years (Engel Class IC). Two patients (16.7\%) had rare disabling seizures following RES (nocturnal only, Engel Class IID). One patient experienced a worthwhile reduction in seizures but was not seizure free (Engel Class III).

Patients rated satisfaction with surgery as excellent $(8$ [72.7\%] of 11), good (2 [7.1\%] of 11, including the patient who underwent a right frontal lesionectomy), or poor (1 [3.6\%] of 11). Nine (90\%, including the patient with frontal lobe epilepsy) of the 10 patients who underwent delayed RES said they would have considered surgery earlier if they had the chance to remake the decision, including 1 patient who was not completely satisfied with her postoperative seizure outcome.

\section{Medical Comorbidities}

The majority of patients (9 [75\%] of 12) had at least 1 preoperative medical comorbidity, including $8(72.7 \%)$ of the 11 patients who underwent temporal resections (Tables 4 and 5). The mean Charlson Comorbidity Index score was $3.7 \pm 1.3$ (range 2-7) among all the patients. The mean score was almost identical when limited to the patients who underwent temporal resections $(3.6 \pm 1.4$, range $2-7)$. The Charlson Comorbidity Index allowed a mean 10-year survival probability of $61.0 \% \pm 26.8 \%$ (range $0-90 \%$ ) to be calculated. Similar 10-year survival probabilities $(61.7 \%$ $\pm 28.0 \%$, range $0-90 \%$ ) were obtained when the analysis was limited to patients with temporal lobe seizures.

Falls and fractures were an important reason to consider surgery in $6(54.5 \%)$ of 11 patients interviewed postoperatively. However, the fall risk remained elevated after surgery, even in seizure-free patients. Seven (63.6\%) of the 11 patients reported postoperative falls and injuries including fractures. The 2 patients suffering only nocturnal seizures had both suffered repeated fall-related injuries that resulted in a fractured arm and hip in 1 patient and lacerations in the other. However, none of these falls with resulting injuries were caused by seizures.

\section{Postoperative Life Fulfillment Scores}

Eleven patients were interviewed and scored for life satisfaction by utilizing the modified LLF tool. Following surgery, the mean LLF score was $26.7 \pm 6$ (range 10-31). The mean score was unchanged when the analysis was

TABLE 3. Preoperative neuropsychological testing results in 9 patients who underwent RES*

\begin{tabular}{|c|c|c|c|c|c|c|c|c|c|c|c|c|}
\hline $\begin{array}{l}\text { Patient } \\
\text { ID }\end{array}$ & $\begin{array}{c}\text { Age at } \\
\text { Testing (yrs) }\end{array}$ & $\begin{array}{c}\text { Yrs of } \\
\text { Education }\end{array}$ & $\begin{array}{l}\text { Sim } \\
\text { Score }\end{array}$ & $\begin{array}{c}\text { BD } \\
\text { Score }\end{array}$ & $\begin{array}{c}\text { OA } \\
\text { Score }\end{array}$ & $\begin{array}{c}\text { DS } \\
\text { Score }\end{array}$ & $\begin{array}{l}\text { BNT } \\
\text { Score }\end{array}$ & $\begin{array}{l}\text { LM I/II } \\
\text { Scores }\end{array}$ & $\begin{array}{l}\text { VPA I/II } \\
\text { Scores }\end{array}$ & $\begin{array}{l}\text { VR I/II } \\
\text { Scores }\end{array}$ & $\begin{array}{l}\text { BDI-II } \\
\text { Score }\end{array}$ & $\begin{array}{l}\text { BAI } \\
\text { Score }\end{array}$ \\
\hline $\mathrm{B} \dagger$ & 69 & 9 & & & & $641 \ddagger$ & & $40 / 522 \S$ & & $45 / 11$ & & \\
\hline C & 63 & 16 & 25 & 50 & $751 \ddagger$ & $371 \ddagger$ & 61 & $91 / 63$ & $75 / 50$ & $43 / 322 \S$ & & \\
\hline$D$ & 73 & 18 & 50 & & 5 & 63 & 63 & $50 / 63$ & $63 / 84$ & ף & (4) & (5) \\
\hline$F$ & 69 & 16 & 63 & 37 & 63 & 25 & 9 & $50 / 9$ & $95 / 75$ & $94 / 83 \S$ & (4) & (1) \\
\hline G & 63 & 18 & 37 & 37 & 16 & 37 & 42 & $64 / 10$ & $5 / 1$ & $10 / 1$ & (21) & (13) \\
\hline $\mathrm{H}$ & 67 & 15 & 95 & 50 & 50 & 37 & 68 & $9 / 25$ & $63 / 63$ & $5 / 16$ & (4) & (8) \\
\hline 1 & 62 & 14 & 95 & 5 & 63 & 37 & 86 & $50 / 50$ & $50 / 50$ & $37 / 63$ & (20) & (20) \\
\hline $\mathrm{J}$ & 63 & $20+$ & $983^{* *}$ & $753^{* *}$ & & 98 & 21 & $50 / 504 \uparrow \uparrow$ & 63/844†† & $25 / 164 \dagger \dagger$ & (2) & (6) \\
\hline L & 60 & 12 & $253^{* *}$ & $163^{* *}$ & & $253^{* *}$ & 37 & $63 / 374 \dagger \dagger$ & $37 / 254 \dagger \dagger$ & $63 / 374 † \dagger$ & (9) & (18) \\
\hline
\end{tabular}

BAI = Beck Anxiety Inventory; BD = Block Design; BDI-II = Beck Depression Inventory, 2nd Edition; BNT = Boston Naming Test; DS = Digit Span; LM = Logical Memory; $\mathrm{OA}=$ Object Assembly; Sim = Similarities subtest of the Wechsler Adult Intelligence Scale; VPA = Verbal Paired Associates; VR = Visual Reproduction.

* All scores are presented as percentile rankings, with the exception of raw scores, which are presented in parentheses. All LM, VPA, and VR scores are from the Wechsler Memory Scale, 3rd Edition (WMS-III), unless otherwise noted. All BD, OA, and DS scores are from the Wechsler Adult Intelligence Scale, 3rd Edition (WAISIII), unless otherwise noted.

† Patient B's primary language was Farsi. Neuropsychological testing was performed in Farsi with use of an interpreter, although norms based on an English-speaking population were used for scoring.

$\ddagger$ Wechsler Adult Intelligence Scale-Revised (WAIS-R).

$\S$ Wechsler Memory Scale-Revised (WMS-R).

II Because of poor performance on the OA subtest of the WAIS-III, Patient D was administered only the Recognition Format of Visual Reproduction I and II subtests. She had a raw score of 2 out of 4 at both the immediate and delayed time points.

** Wechsler Adult Intelligence Scale, 4th Edition (WAIS-IV).

†† Wechsler Memory Scale, 4th Edition (WMS-IV). 
TABLE 4. Preoperative medical comorbidities in 12 older patients at the time of RES

\begin{tabular}{ll}
\hline \multicolumn{1}{c}{ Comorbidity } & No. of Patients (\%) \\
\hline HTN & $3(25)$ \\
\hline Hyperlipidemia & $2(16.7)$ \\
\hline DM & $2(16.7)$ \\
\hline OSA & $1(8.3)$ \\
\hline Osteoporosis & $1(8.3)$ \\
\hline Hypothyroidism & $6(50)$ \\
\hline Anxiety \&/or depression & $5(41.7)$ \\
\hline
\end{tabular}

$\mathrm{DM}=$ diabetes mellitus type 1 or $2 ; \mathrm{HTN}=$ hypertension; OSA = obstructive sleep apnea.

limited to the patients with temporal resections. Eight (72.7\%) noted excellent satisfaction with their RES, with $5(45.5 \%)$ noting postoperative improvements in overall health. The LLF scores tended to be better in patients whose duration of epilepsy was $\leq 30$ years prior to RES $(29 \pm 1.9)$ than in those whose epilepsy was $>30$ years $(24.8 \pm 7.8$; Wilcoxon rank-sum test with continuity correction $\mathrm{W}=24, \mathrm{p}=0.11)$. The LLF scores also tended to be higher in patients with an Engel Class I outcome (8 patients, mean LLF score $28.5 \pm 2.3$ ) versus those with an Engel Class II-IV outcome ( 3 patients, mean LLF score $22 \pm 10.8 ; \mathrm{W}=14.5, \mathrm{p}=0.42$ ). However, the differences in neither of these factors reached statistical significance. Reasons for low LLF scores $(\leq 33.1)$ included falls and fractures, mood swings, feeling socially isolated and/or lacking a close friend, poor general health, cognitive and memory problems, and poor family relationships.

High LLF scores were reported even though no changes occurred in the spheres of driving and working. No patients who were unemployed prior to RES resumed working postoperatively. Only 1 patient who was not driving prior to RES resumed driving after surgery.

\section{Surgical Complications}

No postoperative complications were reported in 9 pa- tients (75\%), even though 7 (77.8\%) of these 9 patients had at least 1 medical comorbidity at the time of RES. One patient $(8.3 \%)$ had a mild complication (left eye swelling) that quickly resolved postoperatively. Two patients (16.7\%) had more significant postoperative complications; 1 patient had postoperative left facial weakness and 1 had a cerebrospinal fluid leak that was subsequently repaired. Both of these patients completely recovered from their postoperative complications.

\section{Discussion}

Our results show that RES can be safe and effective in patients with an age $\geq 60$ years. More than $90 \%$ of patients in our cohort had a good surgical outcome, with $50 \%$ becoming completely seizure free. The majority (75\%) had no postoperative complication, even though most of them had at least 1 medical comorbidity at the time of RES. Improved overall health at the time of follow-up was recorded for $72.7 \%$ of patients. Our series adds to a growing body of literature suggesting that older patients are just as likely to be rendered seizure free by RES and to encounter no greater surgical risk than their younger counterparts. Most of the previous studies have focused on patients over $45-50$ years but younger than 60 . Only 2 published studies have specifically reported on patients 60 years or older.,12 Age ranges over 45 years include upper age limits $>60$ years in only a small number of studies. ${ }^{20,22}$ However, even including patients older than 60 years at the time of RES does not result in a significant reduction in the rate of postoperative seizure freedom or an increase in complication rates. ${ }^{7,16,22}$

In addition to being safe and efficacious, RES also resulted in high life satisfaction in our older cohort. Almost three-fourths of the cohort noted excellent satisfaction with their RES, with almost half noting postoperative improvements in overall health. Liverpool Life Fulfillment scores tended to be higher in patients who underwent RES sooner rather than later ( $\leq 30$ years after seizure onset) and had complete postoperative seizure freedom. Interestingly, we observed high LLF scores in older patients following RES

TABLE 5. Specific preoperative medical comorbidities in 12 older patients

\begin{tabular}{ccccccccccc}
\hline $\begin{array}{c}\text { Patient } \\
\text { ID }\end{array}$ & HTN & Hyperlipidemia & DM & Osteoporosis & Dementia & Hypothyroid & $\begin{array}{c}\text { Anxietyl } \\
\text { Depression }\end{array}$ & OSA & $\begin{array}{c}\text { CCl } \\
\text { Score }\end{array}$ & $\begin{array}{c}10-Y r \\
\text { Survival (\%) }\end{array}$ \\
\hline A & N & N & N & N & N & N & Y & N & 2 & 90.1 \\
\hline B & N & N & N & N & N & Y & Y & N & 3 & 77.5 \\
\hline C & N & N & N & Y & N & N & Y & N & 3 & 77.5 \\
\hline D & Y & N & N & N & N & Y & N & N & 5 & 21.4 \\
\hline E & N & N & N & N & N & N & Y & N & 3 & 77.5 \\
\hline F & N & N & N & N & N & Y & N & N & 3 & 77.5 \\
\hline G & N & Y & Y & N & N & Y & N & Y & 7 & 0.0 \\
\hline H & N & N & N & N & N & N & N & N & 3 & 77.5 \\
\hline I & N & N & N & N & N & Y & Y & N & 3 & 77.5 \\
\hline J & N & Y & N & N & N & Y & N & N & 4 & 53.4 \\
\hline K & Y & N & N & N & N & N & N & N & 4 & 53.4 \\
\hline L & Y & N & Y & N & N & N & N & N & 4 & 53.4 \\
\hline
\end{tabular}

$\mathrm{CCl}=$ Charlson Comorbidity Index. 
even though no significant change occurred in the percentage that could drive and/or work postoperatively. No patients who were unemployed prior to RES sought employment after RES. Only 1 patient who was not driving prior to RES regained driving privileges postoperatively. In younger patients with epilepsy, employment and driving status are often considered to be among the most important aspects of life compromised by uncontrolled seizures. ${ }^{10}$ This has caused previous researchers to specifically examine these 2 factors when attempting to measure psychosocial outcomes following RES. ${ }^{13}$ However, our data suggest that such factors should be given significantly less weight when attempting to measure life fulfillment in older patients following RES. Many older patients with drug-resistant epilepsy may have already retired and have no plans to seek new employment, regardless of whether they achieve seizure freedom or not. Some older patients may have stopped driving for other reasons (for example, changes in vision, slowed reaction time caused by other medical comorbidities, and so forth). The older patients in our cohort failed to see these abilities as either goals of surgery or essential to their independence and identity. Rather, they focused on other positive aspects of surgery, including improvements in safety, seizure control, and medication reduction, when judging the success or failure of RES. In developing future QOL scales for people with epilepsy, it may be important to take age into consideration when judging the weights to ascribe to driving and working.

Another positive aspect of RES that may apply to older patients is a reduction in falls and resulting injuries. Such a benefit goes beyond a mere reduction in seizure-related falls and injuries. Previous research has shown that patients taking antiseizure drugs (especially enzyme inducers) are at increased risk of fractures, particularly when such drug exposure is prolonged. ${ }^{19}$ Fractures may be more likely because of osteoporosis and lower bone mass caused by enzyme-inducing antiseizure drugs. ${ }^{18}$ In addition, certain antiseizure drugs may interact with other medications that older patients are commonly prescribed, altering their serum levels. ${ }^{11}$ This coupled with potential reduced hepatic and renal clearance in the elderly could increase the frequency of toxicity, result in unsteadiness and/or ataxia, and contribute to falls. When successful, RES may allow physicians to successfully reduce or eliminate some or all antiseizure drugs from an older patient's medication regimen. This, in turn, may substantially lower the risk of drug toxicity, osteoporosis, falls, and/or fractures. Such potential benefit deserves further study in larger cohorts of older patients following RES.

Our study was not without limitations. Given the relatively small number of patients with an age $\geq 60$ years who had undergone RES during the study period, our study was not sufficiently powered to demonstrate more subtle statistical significance with many of our findings. We realize that a larger cohort of older patients, possibly through prospective multicenter collaborations, is needed to more definitively determine the improvements to specific QOL domains offered by RES. The retrospective and subjective nature of some QOL question and answer choices may have resulted in reporting bias, with the patients aiming to support the authors. The cohort in our study represents a series of selected patients offered surgery during the study period. We do not have access to data on older patients who were evaluated and subsequently excluded from undergoing RES, and we acknowledge this limitation. Among the 11 patients who underwent temporal resections, only 1 had a conventional left AMTL, with another having a left temporal lesionectomy sparing the mesial structures. There may have been a perceived reluctance to offer dominant temporal lobe resection to older patients; however, there was no deliberate bias. Therefore, the safety and efficacy of RES in older patients with dominant temporal lobe epilepsy cannot be as readily established from our data. Only 1 patient in our cohort underwent an extratemporal resection. Therefore, we cannot positively conclude that extratemporal lobe resective surgery is safe and effective in patients older than 60 years of age. Given that no patients in our cohort underwent intracranial monitoring, we cannot address the safety and/or efficacy of this diagnostic tool in older patients. Although all patients had follow-up for at least 1 year after RES, 4 patients had follow-up that was less than 2 years. Given that we did not administer the LLF tool to our older patients preoperatively, we cannot objectively assess for significant quantitative improvements in life fulfillment in our cohort. However, given that a majority of our patients reported excellent satisfaction with their surgery and would in hindsight undergo surgery sooner, we felt there were probably improvements. Given that neuropsychological testing is not routinely performed at our institution after RES (as many tests are not approved by insurance), we cannot comment on potential postoperative changes on objective measures of verbal and nonverbal memory in older patients.

Despite these limitations, the findings of our study deserve attention. Our cohort is one of the largest to show that older patients beyond the age of 60 benefit from RES. The procedure can be safe and effective and has the potential to increase life fulfillment in all patients regardless of age. Older patients are currently an underrepresented population in the epilepsy literature. It is possible that older patients are more carefully screened simply because of age and may be unjustly denied a life-enhancing treatment. This is particularly pertinent given that this population may experience greater morbidity from seizures and antiseizure medications. Given advances in surgical technique and the advent of newer, less invasive procedures such as real-time MR-guided stereotactic laser thermal ablation, ${ }^{23}$ there are now fewer reasons to withhold this option from older patients. We owe it to all patients to look beyond age when considering the possibility of RES and to expand the demographics of those referred for comprehensive epilepsy evaluations to include the older patient.

\section{Conclusions}

Resective epilepsy surgery can be safe, effective, and satisfying in patients aged 60 years or older. The majority $(91.7 \%)$ of older patients who underwent RES at our institution had a good postsurgical outcome (Engel Class I-II). Half were completely seizure free (Engel Class IA), even though the majority had 1 or more medical comorbidities. Almost $75 \%$ of older patients noted excellent satisfaction 
with their RES, with many (45.5\%) noting postoperative improvements in overall health. Our data demonstrate that advancing age by itself should not prohibit the consideration of RES.

\section{Acknowledgments}

We thank Dror Berel for his assistance with the statistical analysis. Dr. Engel receives research support from the NIH (NINDS P01 NS002808 [PI] and NINDS R37 NS033310 [PI]), the Epilepsy Foundation, and from private donors. Dr. Fried has received an institutional grant from the National Institute of Neurological Disorders and Stroke (Grant Nos. R21 NS37897 and U01 NS42372).

\section{References}

1. Acosta I, Vale F, Tatum WO IV, Benbadis SR: Epilepsy surgery after age 60. Epilepsy Behav 12:324-325, 2008

2. Begley CE, Famulari M, Annegers JF, Lairson DR, Reynolds TF, Coan S, et al: The cost of epilepsy in the United States: an estimate from population-based clinical and survey data. Epilepsia 41:342-351, 2000

3. Boling W, Andermann F, Reutens D, Dubeau F, Caporicci L, Olivier A: Surgery for temporal lobe epilepsy in older patients. J Neurosurg 95:242-248, 2001

4. Cascino GD, Sharbrough FW, Hirschorn KA, Marsh WR: Surgery for focal epilepsy in the older patient. Neurology 41:1415-1417, 1991

5. Charlson M, Szatrowski TP, Peterson J, Gold J: Validation of a combined comorbidity index. J Clin Epidemiol 47:12451251,1994

6. Charlson ME, Pompei P, Ales KL, MacKenzie CR: A new method of classifying prognostic comorbidity in longitudinal studies: development and validation. J Chronic Dis 40:373383, 1987

7. Costello DJ, Shields DC, Cash SS, Eskandar EN, Cosgrove GR, Cole AJ: Consideration of epilepsy surgery in adults should be independent of age. Clin Neurol Neurosurg 111:240-245, 2009

8. Engel J Jr: Surgical Treatment of the Epilepsies. New York: Raven Press, 1993, pp 786

9. Engel J Jr, McDermott MP, Wiebe S, Langfitt JT, Stern JM, Dewar S, et al: Early surgical therapy for drug-resistant temporal lobe epilepsy: a randomized trial. JAMA 307:922-930, 2012

10. England MJ, Liverman CT, Schultz AM, Strawbridge LM: Epilepsy across the spectrum: promoting health and understanding. A summary of the Institute of Medicine report. Epilepsy Behav 25:266-276, 2012

11. Gallo BV: Epilepsy, surgery, and the elderly. Epilepsy Res 68 (Suppl 1):S83-S86, 2006

12. Grivas A, Schramm J, Kral T, von Lehe M, Helmstaedter C, Elger CE, et al: Surgical treatment for refractory temporal lobe epilepsy in the elderly: seizure outcome and neuropsychological sequels compared with a younger cohort. Epilepsia 47:1364-1372, 2006

13. Jones JE, Blocher JB, Jackson DC: Life outcomes of anterior temporal lobectomy: serial long-term follow-up evaluations. Neurosurgery 73:1018-1025, 2013

14. Kotsopoulos IA, van Merode T, Kessels FG, de Krom MC, Knottnerus JA: Systematic review and meta-analysis of incidence studies of epilepsy and unprovoked seizures. Epilepsia 43:1402-1409, 2002

15. McLachlan RS, Chovaz CJ, Blume WT, Girvin JP: Temporal lobectomy for intractable epilepsy in patients over age 45 years. Neurology 42:662-665, 1992

16. Murphy M, Smith PD, Wood M, Bowden S, O'Brien TJ, Bulluss KJ, et al: Surgery for temporal lobe epilepsy associated with mesial temporal sclerosis in the older patient: a longterm follow-up. Epilepsia 51:1024-1029, 2010
17. Sander JW, Hart YM, Johnson AL, Shorvon SD: National General Practice Study of Epilepsy: newly diagnosed epileptic seizures in a general population. Lancet 336:1267-1271, 1990

18. Sheth RD, Harden CL: Screening for bone health in epilepsy. Epilepsia 48 (Suppl 9):39-41, 2007

19. Shiek Ahmad B, Hill KD, O'Brien TJ, Gorelik A, Habib N, Wark JD: Falls and fractures in patients chronically treated with antiepileptic drugs. Neurology 79:145-151, 2012

20. Sirven JI, Malamut BL, O'Connor MJ, Sperling MR: Temporal lobectomy outcome in older versus younger adults. Neurology 54:2166-2170, 2000

21. Smith DF, Baker GA, Dewey M, Jacoby A, Chadwick DW: Seizure frequency, patient-perceived seizure severity and the psychosocial consequences of intractable epilepsy. Epilepsy Res 9:231-241, 1991

22. Srikijvilaikul T, Lerdlum S, Tepmongkol S, Shuangshoti S, Locharernkul C: Outcome of temporal lobectomy for hippocampal sclerosis in older patients. Seizure 20:276-279, 2011

23. Willie JT, Laxpati NG, Drane DL, Gowda A, Appin C, Hao $\mathrm{C}$, et al: Real-time magnetic resonance-guided stereotactic laser amygdalohippocampotomy for mesial temporal lobe epilepsy. Neurosurgery 74:569-585, 2014

\section{Disclosure}

Ms. Dewar previously served on the Professional Advisory Board of the Epilepsy Foundation of America. Dr. Eliashiv serves on advisory boards and speakers bureaus for UCB, the Epilepsy Foundation of America, Sunovion, and Lundbeck. Dr. Eliashiv also receives grant support from DARPA (DARPA-BAA-14-08). Dr. Walshaw has no disclosures. Dr. Engel has received funding for travel or speaker honoraria from Eisai Inc., UCB, Johnson \& Johnson, Novartis, Best Doctors, and S\&S HealthCare Strategies; serves on the editorial boards of Epilepsia, Epileptic Disorders, Brain Stimulation, Experimental Neurology, and WFN Seminars in Clinical Neurology, and as an Associate Editor of MedLink; serves as a consultant for Acorda Therapeutics Inc.; and holds patents regarding Magnetonanoparticle for MRI for which he has received royalty and license fee payments from Epinano, Inc. Dr. Moseley has performed previous consulting work for Nonin Medical, Inc. and serves on the editorial boards for the Journal of Neurology \& Translational Neuroscience, Pediatric and Neonatal Care, and Molecular and Cellular Epilepsy. All authors take part in clinical work that includes workups for potential resective epilepsy surgery. No outside/third party funding was used for this study.

\section{Author Contributions}

Conception and design: Moseley, Dewar. Acquisition of data: Dewar, Walshaw. Analysis and interpretation of data: Moseley, Dewar, Eliashiv, Walshaw. Drafting the article: Moseley, Dewar, Eliashiv, Walshaw. Critically revising the article: Dewar, Eliashiv, Walshaw, Engel, Fried. Reviewed submitted version of manuscript: all authors. Approved the final version of the manuscript on behalf of all authors: Moseley. Statistical analysis: Moseley.

\section{Supplemental Information}

\section{Previous Presentation}

The data in this paper were previously presented in poster form at the American Epilepsy Society 67th Annual Meeting held in Washington, DC, on December 9, 2013.

\section{Correspondence}

Brian D. Moseley, Department of Neurology and Rehabilitation Medicine, University of Cincinnati, 260 Stetson St., Ste. 2300, Cincinnati, OH 45267-0525. email: briandmoseley@gmail.com. 